\title{
A level set method for materials with texturally equilibrated pores
}

\author{
Soheil Ghanbarzadeh ${ }^{\mathrm{a}}$, Marc A. Hesse $\mathrm{b}^{\mathrm{b}, \mathrm{c}, *}$, Maša Prodanovića ${ }^{\mathrm{a}}$ \\ ${ }^{a}$ Department of Petroleum and Geosystems Engineering, The University of Texas at Austin, Austin, Texas 78712 \\ ${ }^{b}$ Department of Geological Sciences, The University of Texas at Austin, Austin, Texas 78712 \\ ${ }^{c}$ Institute for Computational Engineering and Sciences, The University of Texas at Austin, Austin, Texas 78712
}

\begin{abstract}
Textural equilibrium controls the distribution of the liquid phase in many naturally occurring porous materials such as partially molten rocks and alloys, salt-brine and ice-water systems. In these materials, pore geometry evolves to minimize the solid-liquid interfacial energy while maintaining a constant dihedral angle, $\theta$, at solid-liquid contact lines. We present a level set method to compute an implicit representation of the liquid-solid interface in textural equilibrium with space-filling tessellations of multiple solid grains in three dimensions. Each grain is represented by a separate level set function and interfacial energy minimization is achieved by evolving the solid-liquid interface under surface diffusion to constant mean curvature surface. The liquid volume and dihedral angle constraints are added to the formulation using virtual convective and normal velocity terms. This results in a initial value problem for a system of nonlinear coupled PDEs governing the evolution of the level sets for each grain, using the implicit representation of the solid grains as initial condition. A domain decomposition scheme is devised to restrict the computational domain of each grain to few grid points around the grain. The coupling between the interfaces is achieved in a higher level on the original computational domain. The spatial resolution of the discretization is improved through highorder spatial differentiation schemes and localization of computations through domain composition. Examples of three-dimensional solutions are also obtained for different grain distributions networks that illustrate the geometric flexibility of the method.
\end{abstract}

Keywords: Level set method; Textural equilibrium; Percolation; Dihedral angle; Mean Curvature

\section{Introduction}

Textural equilibrium determines the solid-liquid topology in many natural materials, such as partially molten rocks [1], ice-water systems [2], salt-brine systems [3] and alloys [4], see [5] for a recent review. Textural equilibrium is the state of thermodynamic equilibrium where the interfacial area has evolved to minimize the solid-liquid surface energy density, $\gamma_{s l}[6]$, and hence to constant mean curvature, $k$, if the pressure is hysdrostatic and the grains are isotropic. In these materials the topology and geometry of the pore network is controlled by the dihedral angle, $\theta$, which is a function of the surface energies of the mineral grains and the pore fluid [5]. The basic theory of textural equilibrium in two-phase materials have been introduced by (author?) $[4,7]$ in the context of partially molten alloys.

\footnotetext{
${ }^{*}$ Corresponding author

Email address: mhesse@ices.utexas. edu (Marc A. Hesse)
}

Texturally equilibrated pores are common in porous materials with fast solid-liquid kinetics or in cases where long equilibration time scales are available, therefore they are common in geological systems. In most cases, solid-solid interfaces can be considered stationary on the timescale required to reach textural equilibrium of the pore network $[1,5]$, so that the solid-solid surface energy density, $\gamma_{s s}$, is not minimized. Figure 1a illustrates how these pre-existing grain-grain boundaries impose a lattice on the pore space and introduces contact lines along which solid-liquid and solid-solid interfaces meet at sharp angles (Figure 1b). In a two phase material with isotropic surface energies, mechanical equilibrium at the contact line requires that

$$
\gamma_{s s}=2 \gamma_{s l} \cos (\theta / 2)
$$

where $\theta$ is the dihedral angle, $\gamma_{s s}$ and $\gamma_{s l}$ are solid-solid and solid-liquid surface energy density, respectively [6].

The physical principles of textural equilibrium are

May 8, 2015 
similar to standard wetting problems [8]. The most important difference between textural equilibrium and standard wetting problems is the role of the solid. In typical wetting problems the solid geometry is given and does not evolve. In the problems considered here the solid has a dual role. The solid-solid grain boundaries and edges do not evolve and provide a static lattice for fluid percolation. The solid-liquid grain boundaries, however, do evolve and determine the topology percolation of the pore space.

A characteristic of texturally equilibrated porous media is that the pore network percolates at any porosity for $\theta \leq 60^{\circ}$ while a percolation threshold exists for $\theta>60^{\circ}$ [9]. This property is specially important in comparison with the percolation theories in granular porous media where a porosity of $3 \%$ is required for connectivity of the pore space [10]. This ability of texturally equilibrated pore networks to percolate at very low porosities provides an elegant explanation for several geological observations $[11,12,2]$. For example, the small dihedral angle between basaltic melt and olivine explains the near instantaneous extraction of partial melts beneath mid-ocean ridges that was inferred from a number of indirect observations [9, 1, 13, 14, 15]. The decrease of the dihedral angle between rock salt and brine with increasing pressure and temperature $[3,16]$ can explain how rock salt that is generally impermeable at shallow depth [17] can become become permeable and stained by oil at greater depth [12].

The first models which calculated the threedimensional shape of pore networks in textural equilibrium were developed by [18] and [1]. The former was based on interfacial surface energy minimization and the latter was developed based on the idea that at equilibrium, chemical potential of components in dif-

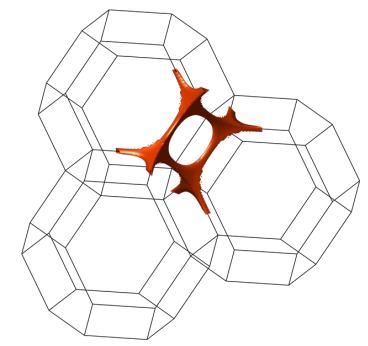

(a)

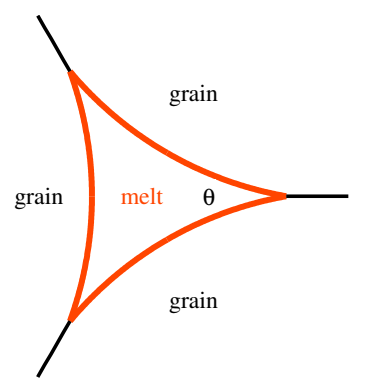

(b)
Figure 1: (a) Wireframe of three truncated octahedron grains with a texturally equilibrated grain edge porosity of $1 \%$. (b) Cross section of a grain-edge channel illustrating the definition of dihedral angle, $\theta$. Images from (author?) [5] used with permission from the American Physical Society. ferent phases is constant. Both models eventually reach to same essential condition for texturally equilibrated pores

$$
\kappa=\text { const }
$$

where $\kappa$ is mean curvature of the solid-liquid interface for a two-phase system under hydrostatic pressure and with isotropic surface energies. Later, the model developed in [1] was reproduced to study the seismic wave velocities of partially molten rocks [19] and their electrical properties [20]. Recently, (author?) [21] developed a three-dimensional micro-geodynamic model to solve for grain-melt geometry in an isotropic unit cell comprised of rhombic dodecahedral grains balancing pressure, surface tension, and viscous deformation forces.

A closed surface which minimizes the area subject to a fixed enclosed volume must have constant mean curvature, $\kappa$ [22]. Therefore, in textural equilibrium, solidliquid interface is a minimal surface subject to dihedral angle condition at boundaries. Considering a solidliquid interface given by $z=f(x, y)$, mean curvature can be defined as

$$
\kappa=\frac{\left(1+f_{y}^{2}\right) f_{x x}+\left(1+f_{x}^{2}\right) f_{y y}-2 f_{x} f_{y} f_{x y}}{2\left(1+f_{x}^{2}+f_{y}^{2}\right)^{\frac{3}{2}}}=\mathrm{const}
$$

which should be constant at textural equilibrium problem and simultaneously, solid-liquid interfaces need to satisfy dihedral angle condition (Eq. 1) at boundaries. In order to close the problem with additional unknown of $\kappa=$ const, Eq. 3 should be solved with volume constraint

$$
V_{f}=\int_{\Omega} f(x, y) d x d y .
$$

The system of equations presented in Eqs. 3 and 4 subject to Neumann boundary conditions is a free boundary problem in Cartesian coordinate system. This equation should be solved coupled with systems representing other disconnected pieces of solid-liquid interfacial surfaces in medium. This results in very complex system which requires topology tracking and changing computational domains. Also free boundary nature of the problem makes it more complicated to be solved in systems with complex grain configurations. Current three-dimensional computational models [including $18,1,23,24]$ are therefore limited by symmetry assumptions in grain geometry resulting in unrealistic 
pore shapes which do not link up to a network in threedimensions.

Despite extensive studies and the common ocurrence of materials with texturally equilibrated pores, first order questions have not been resolved. Of particular importance, is the presence of percolation path and wetted grain faces and their effects on the physical properties of these porous media $[25,19,26]$ in both isotropic and anisotropic crystal lattices [5]. To address this problems we propose a novel level set model to determine an implicit representation of liquid distribution in textural equilibrium with realistic and complicated polycrystalline solids. This model is verified comparing the two-dimensional results with solutions to minimal surface problem. Two-dimensional representation of Eq. 3 in cylindrical coordinate system, $r=f(\Theta)$, is given by

$$
\kappa=\frac{r^{2}+2 r^{\prime 2}-r r^{\prime \prime}}{\left(r^{2}+r^{\prime 2}\right)^{\frac{3}{2}}}=\text { const }
$$

which removes the free boundary nature of problem. Here $r^{\prime}$ and $r^{\prime \prime}$ are first and second derivatives with respect to $\Theta$.

The level set method [27, 28] is a numerical technique that tracks a surface by representing it as a zero level set of a hypersurface, $\varphi$. This allows propagating the interface by solving an initial value problem for the evolution of $\varphi$ governed by a Hamilton-Jacobi equation. In this setting, curvatures and normals may be evaluated easily and topological changes occur in a natural manner [29] and thus it is a good choice for modeling the behavior of complex surfaces. As the method uses a fixed Cartesian grid, extension to any number of dimensions is straightforward. The level set function, $\varphi$, separates the interior and exterior region of an interface by its sign and evolves by general equation of the form

$$
\varphi_{t}+\mathbf{v} \cdot \nabla \varphi=0
$$

where $\mathbf{v}$ is the interface velocity and includes the physics of problem discussed in Section 2. While a level set function undergoes the evolution with appropriate velocities, it is important to keep the magnitude of its gradient $(|\nabla \varphi|)$ bounded to ensure the numerical stability, convergence and accuracy. To do so, the level set function is replaced by a signed distance function in a process called reinitialization that does not move the interface position. To reinitialize $\varphi$, we solve

$$
\varphi_{t}+\operatorname{sign}(\varphi)[|\nabla \varphi|-1]=0
$$

every few time step [30]. This process diffuses the interface in sub-resolution scales and corrections to the method exist in [31]. The gradient magnitude of the steady-state answer to Eq. 7 is one in computation domain.

The incorporation of solid-liquid contact angle is an important application in multiphase flow simulations [32, 33, 34]. Most of these studies consider a flow of single droplet on flat surfaces and the contact angle condition is applied by local reconstruction of level set function during reinitialization. (author?) [35] extended the level set formulation of the critical displacements of fluids during drainage and imbibition in porous media [36] to include the solid-liquid contact angle. They combined the fluid equation of motion in the pore space [36] and fluid contact angle term at the solid phase [37] into a single evolution equation over the entire computational domain.

In this work we follow a similar procedure to include the dihedral angle on solid-liquid contact lines. But here we need two level set functions for each grain, $i$, one representing solid grain, $\psi_{i}$, and one representing liquid phase around the same grain, $\varphi_{i}$. Surface diffusion happens in many film growth problems and the steadystate solution to interface motion driven by surface diffusion is a constant mean curvature surface [38, 31]. Therefore, in order to obtain constant mean curvature surface, the solid-liquid interface evolves with surface Laplacian of curvature as interface speed. This evolution is volume conservative so the desired volume of liquid (porosity) is achieved by adding a normal velocity term to the equation that inflates or deflates $\varphi$. The dihedral angle condition is added to the formulation in form of normal and convective velocity terms, which adjust the interface on solid-liquid contact lines. The solid-liquid contact line forms in places where two adjacent solid grains and their associated liquid level sets meet.

The resulting system of nonlinear PDEs is solved explicit in time with an implicit representation of grains as initial guess. The simulation performance is optimized by computational domain decomposition for each grain $\left(\Omega_{i}\right)$ and evaluating coupling terms on the original computational grid $(\Omega)$. The domain decomposition is used to limit the computational domain size of each grain to the region outside and close to the interface. The method is tested by demonstrating a narrow distribution of mean curvature on solid-liquid interface and comparing the distribution of steady state dihedral angles with the prescribed angle. Unlike previous methods [1, 18] the developed method is not limited by grain geometry constraints. The geometric flexibility of the method is 
demonstrated by presenting pore geometry in isotropic and anisotropic textures with equal and unequal grains as simulation examples.

\section{Level Set Formulation}

Assuming hydrostatic pressure and isotropic surface energy densities, the solid-liquid interface must satisfy both conditions stated in Eq. 1 and 2 simultaneously [1, 9]. Surfaces of constant mean curvature are given by the steady-state solutions to interface motion driven by surface diffusion $[38,31]$. The velocity of an interface moving with surface diffusion is the surface Laplacian of curvature, $\Delta_{s} \kappa$. Then the evolution equation takes the form

$$
\varphi_{t}+\Delta_{s} \kappa|\nabla \varphi|=0
$$

in which $\varphi$ is a level set function and the interface is given by its zero level-set. The interface given by steady-state answer of Eq. 8 is a constant mean curvature which preserves the volume [31]. The surface Laplacian of curvature is given by

$$
\Delta_{s} \kappa=\nabla_{s} \cdot \nabla_{s} K
$$

where

$$
\nabla_{s}=(\nabla-\vec{n}(\vec{n} \cdot \nabla)),
$$

and $\vec{n}$ is the outward normal and $\kappa$ is the mean curvature

$$
\kappa=\nabla \cdot \vec{n}=\nabla \cdot\left(\frac{\nabla \varphi}{|\nabla \varphi|}\right)
$$

Function $\varphi$ separates the domain $(\Omega)$ to interior $\left(\Omega^{-}\right)$ and exterior $\left(\Omega^{+}\right)$regions. Mathematically, we represent this by smoothed Heaviside function

$$
H(\varphi)=\left\{\begin{array}{lr}
0 & \varphi<-\epsilon \\
\frac{1}{2}+\frac{\varphi}{2 \epsilon}+\frac{1}{2 \pi} \sin \left(\frac{\pi \varphi}{\epsilon}\right) & -\epsilon \leq \varphi \leq \epsilon \\
1 & \varphi>\epsilon
\end{array}\right.
$$

where $1.5 \Delta x \leq \epsilon \leq 3 \Delta x$ is a tuning parameter. The interface can be identified by $\delta(\varphi)$ function which is the derivative of $H(\varphi)$.

In the method presented here, each grain, $i$, is represented by two level sets, one representing the solid-solid interfaces of the grain, $\psi_{i}$, and another representing the solid-liquid interfaces of the grain, $\varphi_{i}$. The level-set for the stationary soild-solid grain boundaries does not evolve in time and provides a reference frame for the solid-liquid contact lines. To compute the solid-liquid interface with constant mean curvature, the $\varphi_{i}$ level set evolves by surface diffusion. Due to computational difficulties and instability from unbounded values of $\Delta_{s} \kappa$ [38], we only consider surface diffusion close to interface, in the region where $\delta(\varphi)$ is nonzero. Then, in a polycrystalline material with $N$ grains, the liquid level set of the $i$-th grain undergoes evolution with

$$
\left(\varphi_{i}\right)_{t}+\left[\hat{\delta}\left(\varphi_{i}\right) H\left(-\psi_{i}\right)\right] \Delta_{s} \kappa_{i}\left|\nabla \varphi_{i}\right|=0
$$

The term $\hat{\delta}\left(\varphi_{i}\right)=\Delta x \delta\left(\varphi_{i}\right)$, where $\Delta x$ is the grid size, avoids large values of the delta function $(\delta \propto 1 / \Delta x)$. The term $H\left(-\psi_{i}\right)$ ensures that the diffusive motion is occurring inside and close to interface of each individual grain.

The other constraints of the problem, the desired porosity and the dihedral angle on solid-liquid contact lines, must be added to formulation as additional terms to Eq. 13. In each time step, the global implicit function representing the solid-liquid interface as its zero level set, $\Phi$, on the original computational domain $(\Omega)$ is computed as union of $\varphi_{i}$ 's by

$$
\Phi=\min \left(\varphi_{1}, \varphi_{2}, \ldots, \varphi_{N}\right),
$$

because $\varphi_{i}$ is positive outside the $i$-th grain. The liquid volume in each time step is then calculated using

$$
V_{f}=\int_{\Omega} H(-\Phi) d V
$$

which results in $\mathrm{O}(\Delta x)$ volume accuracy regardless of the integration method used [28]. The relative error between current and desired volume, $\hat{v}$, can then easily be evaluated. In order to adjust the porosity, a normal motion is added to the evolution equation of the level set representing the solid-liquid interfaces, $\varphi_{i}$. This motion causes the liquid phase to expand or shrink by moving the level set function in a normal direction. The velocity of normal motion is proportional to $\hat{v}$ and is scaled by factor of $\Delta x^{-2} e^{\hat{v}}$, by trial and error, in order to be comparable to other terms in Eq. 13. This velocity term vanishes as porosity of medium converges to desired value. Eq. 13 then can be rewritten as follow

$$
\left(\varphi_{i}\right)_{t}+\left[\hat{\delta}\left(\varphi_{i}\right) H\left(-\psi_{i}\right)\right] \Delta_{s} \kappa_{i}\left|\nabla \varphi_{i}\right|+\left[\frac{\hat{v}}{\Delta x^{2}} e^{\hat{v}}\right]\left|\nabla \varphi_{i}\right|=0
$$

The other constraint of the problem which must be satisfied is the mechanical equilibrium condition along the liquid-solid-solid contact lines. The liquid region is defined by the intersection of the outside of all liquid level sets associated to each solid grain $(\varphi)$, mathematically represented by Eq. 14. As can be seen in Fig. 2, 


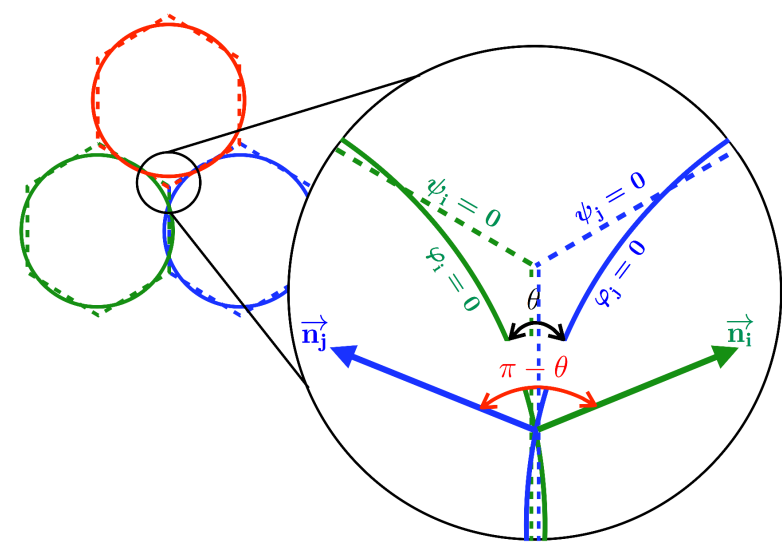

Figure 2: Two-Dimensional description of the dihedral angle, dihedral edge, liquid and solid level sets. While the two corresponding liquid level sets, $\varphi_{i}$ and $\varphi_{j}$, meet with the angle $\theta$, their normals make the angle $\pi-\theta$ with each other.

two liquid level sets $\left(\varphi_{i}\right.$ and $\left.\varphi_{j}\right)$ intersect with dihedral angle $\theta$ on corresponding grain faces and the normals to interface make the angle $\pi-\theta$ with each other [35]. Satisfying the dihedral angle condition

$$
\left[\vec{n}_{i} \cdot \vec{n}_{j}-\cos (\pi-\theta)\right]\left[\hat{\delta}\left(\varphi_{i}\right) \hat{\delta}\left(\varphi_{j}\right)\right]\left[\hat{\delta}\left(\psi_{i}\right) \hat{\delta}\left(\psi_{j}\right)\right]=0
$$

The term $\hat{\delta}\left(\varphi_{i}\right) \hat{\delta}\left(\varphi_{j}\right)$ ensures that the dihedral angle condition is only active where two level set meet and term $\hat{\delta}\left(\psi_{i}\right) \hat{\delta}\left(\psi_{j}\right)$ keeps the intersection point on grain-grain contact between grains $i$ and $j$. Substituting the definition of the interface normal, $\vec{n}=\nabla \varphi /|\nabla \varphi|$, Eq. 17 can be expressed as

$$
\begin{aligned}
& {\left[\nabla \varphi_{i} \cdot \nabla \varphi_{j}-\left|\nabla \varphi_{i}\right|\left|\nabla \varphi_{j}\right| \cos (\pi-\theta)\right]} \\
& {\left[\hat{\delta}\left(\varphi_{i}\right) \hat{\delta}\left(\varphi_{j}\right)\right]\left[\hat{\delta}\left(\psi_{i}\right) \hat{\delta}\left(\psi_{j}\right)\right]\left[S\left(\varphi_{i}\right) S\left(\varphi_{j}\right)\right]=0}
\end{aligned}
$$

in which $S(\varphi)$ is the sign function suggested by (author?) [30] and $S\left(\varphi_{i}\right) S\left(\varphi_{j}\right)$ term enforces the dihedral angle to spread away from adjacent level sets and secures the numerical stability [35]. Eq. 18 contains two velocity terms for the motion of $i$-th level set, one normal and one convective, that move the interface at liquid-solid-solid edges to satisfy dihedral angle condition. The final equation for evolution of the $i$-th level set is obtained by combining Eq. 16 and 18, so that

$$
\begin{aligned}
&\left(\varphi_{i}\right)_{t}+ {\left[\hat{\delta}\left(\varphi_{i}\right) H\left(-\psi_{i}\right)\right] \Delta_{s} \kappa_{i}\left|\nabla \varphi_{i}\right| } \\
&+ {\left[\frac{\hat{v}}{\Delta x^{2}} e^{\hat{v}}-\cos (\pi-\theta) \hat{\delta}\left(\varphi_{i}\right) \hat{\delta}\left(\psi_{i}\right) S\left(\varphi_{i}\right)\right.} \\
&\left.\sum_{j=1: n}^{j \neq i}\left\{\hat{\delta}\left(\varphi_{j}\right) \hat{\delta}\left(\psi_{j}\right) S\left(\varphi_{j}\right)\left|\nabla \varphi_{j}\right|\right\}\right]\left|\nabla \varphi_{i}\right| \\
&+ {\left[\hat{\delta}\left(\varphi_{i}\right) \hat{\delta}\left(\psi_{i}\right) S\left(\varphi_{i}\right)\right.} \\
&\left.\sum_{j=1: n}^{j \neq i}\left\{\hat{\delta}\left(\varphi_{j}\right) \hat{\delta}\left(\psi_{j}\right) S\left(\varphi_{j}\right) \nabla \varphi_{j}\right\}\right] \cdot \nabla \varphi_{i}=0 \\
& \text { for } \quad i=1 \ldots N
\end{aligned}
$$

where $N$ is the number of grains and Eq. 19 needs to be solved for each grain, thus it represents a system of $N$ coupled non-linear PDEs. Textural equilibrium is achieved once the steady-state solution to Eq. 19 is obtained. In steady-state, the constant mean curvature of the solid-liquid interface will result in zero values for $\Delta_{s} \kappa \hat{\delta}\left(\phi_{i}\right)$, the term $\hat{v}$ will be zero and satisfaction of Eq. 17 returns normal and convective velocities which cancel out each other. In order to keep the level set a signed distance function and preserve numerical stability, one needs to solve Eq. 7 every few time steps. As the intersection of level sets creates the actual liquid distribution, final level set representing texturally equilibrated pore network with dihedral angle, $\theta$, and porosity, $\phi$, is then given by Eq. 14 .

\section{Implementation}

The numerical discretization of Eq. 7 and Eq. 19 is implemented in MATLAB ${ }^{\circledR}$ utilizing the Level Set Toolbox developed by (author?) [39]. The toolbox is modified to handle any number of level sets with structure data type, generate grid optimally and evaluate the required velocity terms. Surface Laplacian of curvature $\left(\kappa_{s S}\right)$ is approximated with forth order $\left(\mathrm{O}\left(\Delta x^{4}\right)\right)$ central differences while convective and normal terms are discretized using fifth order $\left(\mathrm{O}\left(\Delta x^{5}\right)\right)$ Hamilton-Jacobi Weighted Essentially Non-Oscillatory (WENO) scheme [28]. Explicit time integration requires a very restrictive CFL condition, $\Delta t<C \Delta x^{4}$, due to the fourth order spatial derivatives in Eq. 19. The constant $C$ is determined in a real-time manner. High-order discretization schemes enable us to use large grid sizes while reducing 
truncation error. In addition to decreasing memory usage, larger grid size results in larger time step required for stability $\left(\Delta t \propto \Delta x^{4}\right)$.

All the boundary conditions are set to bi-linear extrapolation, and numerical discretization on boundaries is done by adding one stencil to computational domains, $\Omega$ and $\Omega_{i}$, in each direction. Time variable in Eq. 19 does not have a physical dimension, $\theta$ and $\hat{v}$ are dimensionless, and delta, Heaviside and sign functions return non-dimensional values. As all the parameters in Eq. 19 are dimensionless, the grid size, $\Delta x$, does not have a physical meaning and it should be compared to grain size. Therefore, $\Delta x$ in simulations is set in a way that a certain number of grid points $\left(N_{\text {grid }}\right)$ span characteristic length of the grain $\left(l_{c}\right)$. All level set functions are also signed distance functions and their values are relative to the grain size.

For each liquid level set function $\left(\varphi_{i}\right)$, simulation starts with implicit representation of the corresponding grain (details in section 3.1) and solution is advanced explicitly in time. All the derivatives and coupled velocity terms are calculated from last time step, then all $\varphi_{i}$ 's undergo evolution with corresponding updates. The simulation continues until the required conditions for textural equilibrium, Eqs. 1 and 2, are satisfied and final porosity is equal to the desired porosity. As the value of mean curvature in final answer is unknown, the error in the curvature is measured by standard deviation in values of curvature while the porosity and dihedral angle are the problem's input and the error in each time step is evaluated as an absolute error. We investigate the validity of these conditions once the steady state answer is reached. An array representing the mean curvature of solid-liquid interface corresponding to grain $i$ is given by

$$
\boldsymbol{\kappa}_{i}=\frac{\kappa_{\varphi_{i}} \hat{\delta}\left(\varphi_{i}\right) H\left(-\psi_{i}\right)\left|\nabla \varphi_{i}\right|}{\hat{\delta}\left(\varphi_{i}\right) H\left(-\psi_{i}\right)\left|\nabla \varphi_{i}\right|} \quad \text { where } \quad \hat{\delta}\left(\varphi_{i}\right) H\left(-\psi_{i}\right) \neq 0
$$

At the end of each iteration, uniformity of mean curvature can be examined by the value of standard deviation $(\sigma)$ in distribution of $\boldsymbol{\kappa}_{i}$ for all grains satisfying

$$
\sigma\left(\kappa_{i}\right) \leq \epsilon_{\kappa} \quad \text { for } \quad i=1 \ldots N
$$

in which $\epsilon_{K}$ is tolerance for variation in value of mean curvature in nodes close to solid-liquid interface. The second condition that needs to be satisfied is mechanical equilibrium at the dihedral edges given by Eq. 1. The error in dihedral angle on solid-liquid contact lines is calculated via

$\mathbf{e}_{\theta_{i}}=\frac{\sum_{j=1: N}^{j \neq i} A_{i j} \hat{\delta}\left(\varphi_{i}\right) \hat{\delta}\left(\varphi_{j}\right)\left|\nabla \varphi_{i}\right|}{\sum_{j=1: N}^{j \neq i} \hat{\delta}\left(\varphi_{i}\right) \hat{\delta}\left(\varphi_{j}\right)\left|\nabla \varphi_{i}\right|}$ where $\sum_{j=1: N}^{j \neq i} \hat{\delta}\left(\varphi_{i}\right) \hat{\delta}\left(\varphi_{j}\right) \neq 0$

where

$$
A_{i j}=\frac{\nabla \varphi_{i}}{\left|\nabla \varphi_{i}\right|} \cdot \frac{\nabla \varphi_{j}}{\left|\nabla \varphi_{j}\right|}-\cos (\pi-\theta)
$$

where $\mathbf{e}_{\theta_{i}}$ is an array containing error in dihedral angle. We assume Eq. 1 is satisfied on solid-liquid contact lines once

$$
\left\|\mathbf{e}_{\theta_{i}}\right\|_{\infty} \leq \epsilon_{\theta} \quad \text { for } \quad i=1 \ldots N
$$

is valid. Here $\|\ldots\|_{\infty}$ denotes infinity norm and $\epsilon_{\theta}$ is the acceptable tolerance for error in dihedral angle.

\subsection{Initialization}

The numerical method requires level set representation for each grain $\left(\psi_{i}\right)$ as input. Solid grains can have arbitrary shape and size, but grain-grain contact is necessary to establish solid-liquid contact lines to apply the dihedral angle constraint. Initial grain representation can optimally be constructed from pore-scale microtomographic images of synthesized or natural samples. To do this, a watershed algorithm should be applied to segmented images to separate the grains. Then signed distance function can be calculated to establish implicit representation of grains. Because the grain separation of the different solid grains is not a trivial process, in this work we have built level set representation of space-filling tessellations in three-dimensional space using signed distance of grid points from grain faces.

In order to create a level set representation for liquid phase corresponding to each grain $\left(\varphi_{i}\right)$, we initialize $\varphi_{i}$ with $\psi_{i}$, then evolve the liquid phase level set $(\varphi)$ with curvature and normal motion terms to round the initial flat liquid interface, establish solid-liquid contact lines instead of contact plains, and to initiate porosity in domain. Therefore, the following evolution equation should be solved establish initial guess for $\varphi_{i}$

$$
\left(\varphi_{i}\right)_{t}+\left(\left(v_{n}\right)_{i}-\kappa_{i}\right)\left|\nabla \varphi_{i}\right|=0 \quad i=1 \ldots N
$$

which needs to be reinitialized (Eq. 7) every few time steps. In this work, the reinitialization processes are done every 5 time steps. The final answer also undergoes reinitialization to establish initial condition of Eq. 19 as a signed distance function. Normal velocity $\left(v_{n}\right)$ 
and final time to stop evolution in Eq. 25 are trivial and $\kappa$ is curvature given by Eq. 11. If a series of simulations with same grain network configuration is being done for a set of $\phi$ or $\theta$ values, this initialization from the grain geometry is only necessary for the first case. Computations can continue using results of previous simulations as initial condition.

\subsection{Domain Decomposition}

The system of non-linear PDEs given by Eq. 19 is coupled though the normal and convective velocities arising from the dihedral angle constraints. The desired porosity is set by a normal velocity which is calculated on entire domain by Eq. 15 and the term which initiate from mean curvature constraints is evaluated independently for each grain. As we are interested in behavior of solid-liquid interface (zero level set), the solution domain can be decomposed for each grain to just include the grain, solid-liquid interface and few grid points away from interface. The coupling between grains can be established on the original computational domain, $\Omega$. To do so, implicit representation of each grains can be separated to inside and outside with logical operations then we can extend the computational domain of each grain to a number of grid points around the grain faces. In this study, we extended the domain from grain faces by $5 \Delta x$ in all directions. Figure 3 shows how initial representation of each grain on $\Omega$, is reduced to smaller sub-domains, $\Omega_{i}$. This method can be applied to any signed distance function representing the grain interface as its zero level set.

At each time step, the coupling velocity terms, originating from the dihedral angle constraint, are reevaluated on $\Omega$ and then mapped yo $\Omega_{i}$. Localization

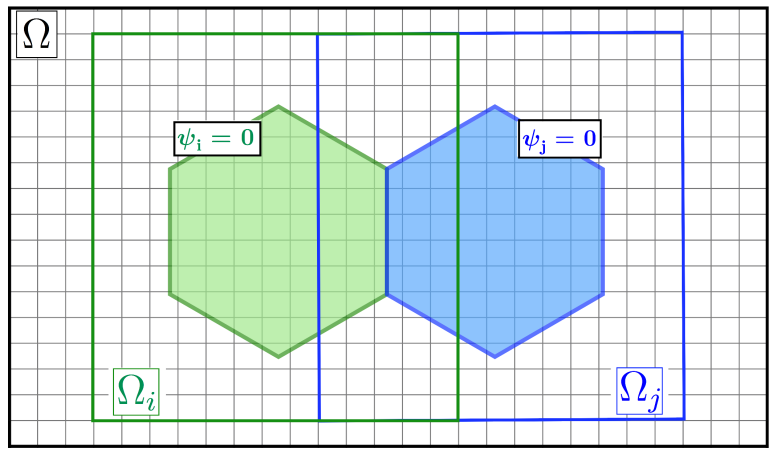

Figure 3: Two-dimensional schematic of domain decomposition Computational domain of each grain, $\Omega_{i}$, is a subset of main computational domain, $\Omega$. Coupling terms between PDEs, which initiate from dihedral angle constraint, are calculated on $\Omega$ and then mapped on $\Omega_{i}$. of computations and domain decomposition reduces the computational cost by orders of magnitude and enables us to solve large complex system presented in Eq. 19 very efficiently, even with a desktop or notebook.

\subsection{Mesh Refinement for Visualization}

Using large grid size with high-order discretization schemes to decrease the numerical error results in low quality visualization of the results. The visualization problem is more severe in three-dimensional simulations due to surface triangulation in the marching cube algorithm [40]. In order to overcome this problem, a mesh refinement scheme is applied to steady-state answer of Eq. 19 and the final level set, representing the liquid-solid interface given by Eq. 14, is interpolated onto a refined grid using a spline gridded interpolation function for each liquid level set, $(\varphi)$.

Fig 4 shows the effect of mesh refinement on visualization of the final results in a network of truncated octahedron grains for two dihedral angles $\theta=30^{\circ}$ and $90^{\circ}$. Fig. $4 \mathrm{a}$ and $4 \mathrm{~d}$ represents the zero-isosurface of the final level set, $\Phi$, with unrefined grid, $N_{\text {grid }} / l_{c}=20$. On the coarse computational grid the triangulation of the interface generated by the marching cube algorithm is not smooth. Fig. 4b and 4e present the zero level set of refined $\Phi$ with two-times refinement $\left(N_{\text {grid }} / l_{c}=40\right)$ and Fig. $4 \mathrm{c}$ and $4 \mathrm{f}$ are generated with five-times refinement $\left(N_{\text {grid }} / l_{c}=100\right)$. Mesh refinement can also improve the accuracy of volume calculation (Eq. 15). Table 1 summarizes the obtained porosity at the end of simulation for visualized cases in Fig. 4. In all simulations,

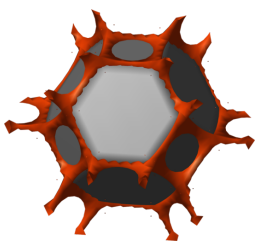

(a)

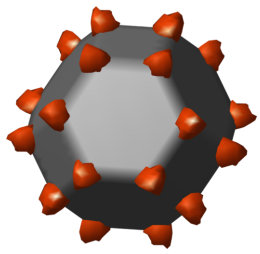

(d)

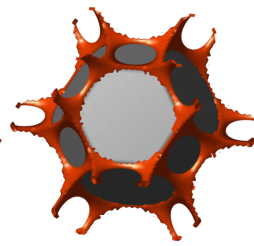

(b)

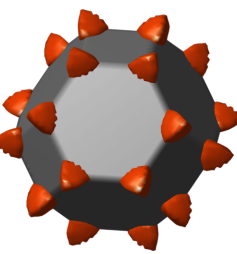

(e)

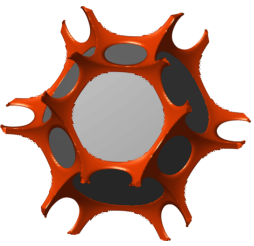

(c)
Figure 4: Comparison of visualization of final level set results with different mesh refinement levels for a case of truncated octahedron grain with $\phi=2 \%$. Original simulation is done with $N_{\text {grid }} / l_{c}=20$. (a-c) $\theta=30^{\circ}$ and $N_{\text {grid }} / l_{c}=20,40$ and 100 from left to right, (d-f) $\theta=90^{\circ}$ and $N_{\text {grid }} / l_{c}=20,40$ and 100 from left to right 
relative error between current and target liquid volume $(\hat{v}$, used in Eq. 19) is calculated using refined $\Phi$ with $N_{\text {grid }} / l_{c}=100$.

Table 1: Final porosity and error between target and obtained porosity with original grid size, $N_{\text {grid }} / l_{c}=20$ and mesh refinement with $N_{\text {grid }} / l_{c}=40$ and 100. Final results are plotted in Fig. 4 . The target volume in all the cases is $2 \%$.

\begin{tabular}{|l|ccc|ccc|}
\hline$\theta$ & \multicolumn{3}{|c|}{$30^{\circ}$} & \multicolumn{3}{c|}{$90^{\circ}$} \\
$N_{\text {grid }} / l_{c}$ & 20 & 40 & 100 & 20 & 40 & 100 \\
\hline$\phi(\%)$ & 2.45 & 2.12 & 1.99 & 2.31 & 2.25 & 2.14 \\
error $(\%)$ & 22.5 & 5.93 & 0.7 & 15.3 & 12.5 & 6.93 \\
\hline
\end{tabular}

\section{Model Verification}

The accuracy of the proposed level-set method for textually equilibrated liquid-solid interface is demonstrated by comparison with analytic solutions in twodimensions. In three dimensions no analytic solutions are available, instead we demonstrate that the numerical solution satisfies the constraints on curvature, dihedral angle, and porosity.

\subsection{Two-Dimensional Simulation}

In two dimensions the problems simplifies greatly, because all constant curvature surfaces are segments of

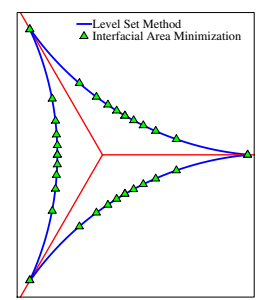

(a)

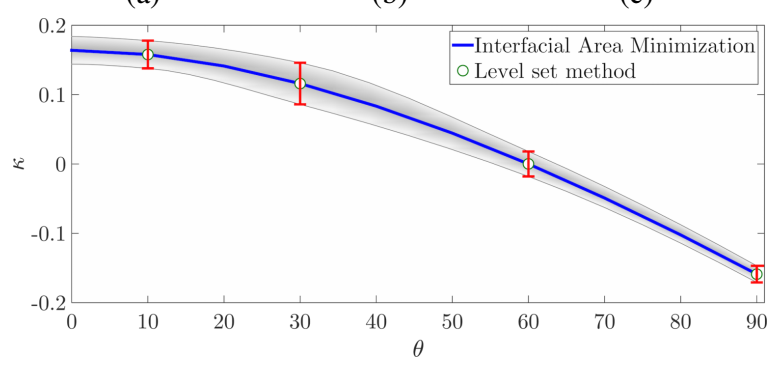

(d)

Figure 5: Effect of $\theta$ on the equilibrated geometry of a twodimensional single pore. Calculations are done using level set method and interfacial area minimization. (a) $\theta=10^{\circ}$, (b) $\theta=30^{\circ}$, (c) $\theta=90^{\circ}$. (d) Comparison of mean curvature of the solid-liquid interface obtained from level set method and interfacial area minimization. The porosity is $10 \%$ in simulations. either circles or lines. The results of proposed method can therefore be compared to the solution of Eq. 5. This equation is essentially a nonlinear ODE and is solved on one-dimensional grid with high-order Newton-Raphson method. Figs. 5b-5c show the equilibrium geometry of a single two-dimensional pore at a symmetric triplejunction at constant $\phi$ for increasing $\theta$ obtained from the proposed level set method and interfacial area minimization. Matching of results verifies the validity of 00 constraints in steady-state answer of the developed nu2.14 merical method. Fig. 5d compares the mean curvature of the solid-liquid interface obtained from both method. The level set method simulations are done for four dihedral angle values and gray shaded area shows the standard deviation in values of mean curvature near the interface. Comparison between the computed shapes in Figs. $5 b-5 c$ and the standard deviation of the curvature in Fig. 5d shows that the standard deviation does not reflect the accuracy of the interface. The distribution in curvatures is mostly due to sampling a curvature in a finite region around the interface.

Fig. 5 confirms that the developed level set model converges to semi-analytical solution in twodimensions. The error in mean curvature value and dihedral angle is very small in two-dimensions and the statement is true even with less number of grid points per grain. In this work all the simulations are done with $N_{\text {grid }} / l_{c}=20$, where $l_{c}$ in all the cases is kept 2 and $\Delta x=0.1$.

\subsection{Three-Dimensional Simulation}

No analytic solution for texturally equilibrated pores is available in three dimensions. Similarly the comparison with previous work is difficult because the source codes are not available. The validation of the three dimensional results is therefore limited to demonstrating that the computed interface has constant curvature and that the constraints on the dihedral angle and porosity are satisfied.

Fig. 6 presents the equilibrated pore geometry in least symmetric sub-volume element in a texturally equilibrated material comprised of truncated octahedron grains. Close inspection of Fig. 6 shows that the 4grain junctions formed by truncated octahedron does not have tetrahedral symmetry that is assumed to simplify the computations in previous works [1, 18, 19]. For isotropic and near isotopic network of grain, change of dihedral angle from $0^{\circ}$ to $60^{\circ}$ has moderate effect on shape of pores. Furtherer change in dihedral angle $\left(>70^{\circ}\right)$ flips the sign of curvature on solid-liquid interface and changes the geometry of the equilibrated pore space dramatically. 


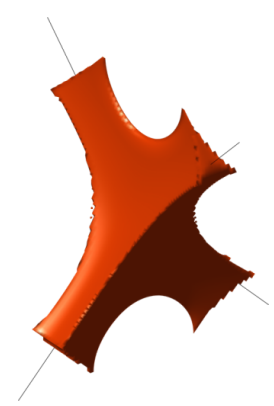

(a)

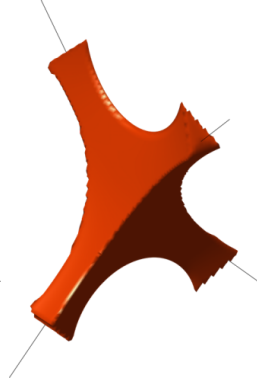

(b)

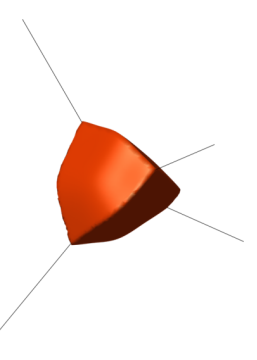

(c)
Figure 6: Effect of $\theta$ on the equilibrium pore geometry at a junction formed at the intersection of four truncated octahedral grains. The visualized pore space is cut from a network of $6 \times 6 \times 6$ grains, with $3 \%$ porosity (a) $\theta=10^{\circ}$, (b) $\theta=30^{\circ}$, (c) $\theta=90^{\circ}$.

Normalized distribution of mean curvature near solid-liquid interface for five conditions with same porosity $(\phi=3 \%)$ and different dihedral angles are plotted in Fig. 7a. The data is collected from a network of $6 \times 6 \times 6$ isotropic truncated octahedron grains. As the total number of grid points near interface is different in each case, the distribution is normalized by the total number of data points and a Gaussian fit is plotted along with data. The situation is very similar to curvature measurements from experimentally detected liquid-liquid interface data [41]. Fig. 7a also shows the distribution of curvature near solid-liquid interface for an anisotropic network of grain, with $\phi=3 \%, \theta=10^{\circ}$ and $f=1.5$. Here $f$ is the elongation factor, by which the grains are stretched in z-direction in order to construct anisotropic grains from initial truncated octahedron grains.

Fig. 7b shows distribution of dihedral angle for similar case studies in Fig. 7a. In all cases the mean of the dihedral angle distribution is within $2^{\circ}$ of the prescribed angle. Figs. 7c-7e the equilibrium interface for cases corresponding to letters (c), (d) and (e) in Fig. 7a. The solid liquid interface is colored by interpolated curvature value which illustrates that the curvature at the equilibrium interface is essentially uniform. Figs. $7 \mathrm{f}$ and $7 \mathrm{~g}$ present the effect of grid size on distribution of curvature and dihedral angle for a case study with $\phi=2 \%$ and $\theta=30^{\circ}$. Refinement reduces the distribution of the values but does not affect the mean values. This suggests that even computations with the coarsest grid give the correct interface. Error bars in Figs. $7 \mathrm{f}$ and $7 \mathrm{~g}$ are derived from standard deviation of fitted Gaussian curves to normalized distribution of curvature and dihedral angle data. As time step required for stability of numerical simulation reduces with using smaller grid

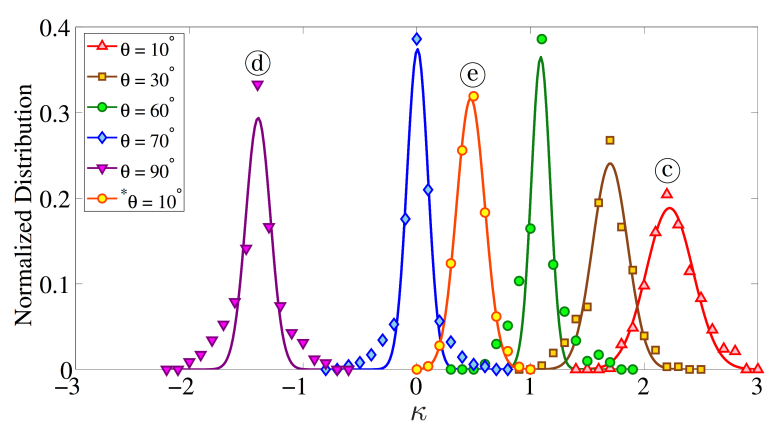

(a)

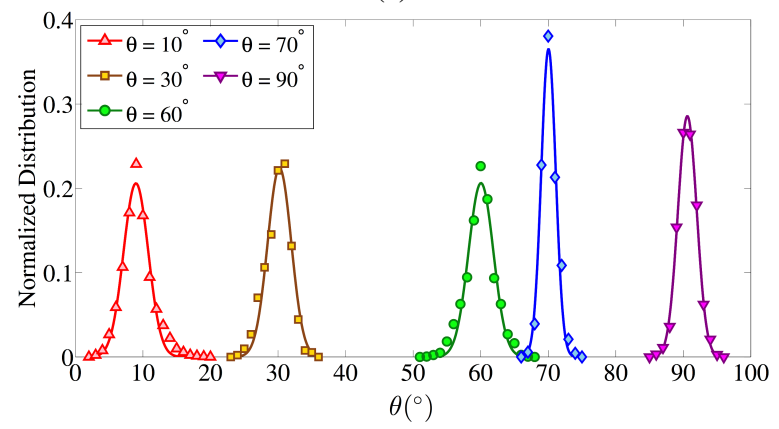

(b)

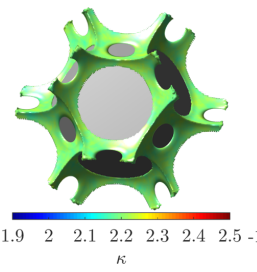

(c)

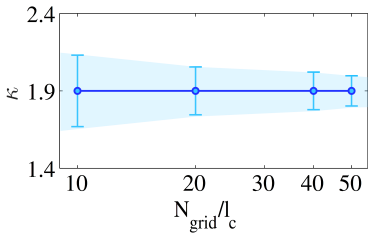

(f)

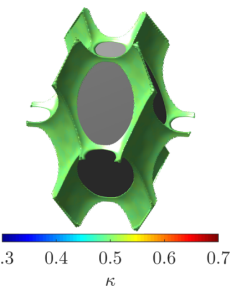

(e) (d)

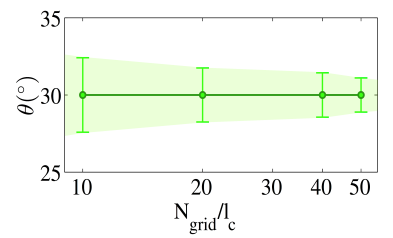

(g)
Figure 7: (a-b) Distribution of mean curvature $(\kappa)$ and dihedral angle $(\theta)$ around solid-liquid interface in three-dimensional simulations. A normal distribution function (Gaussian) is fitted to each data set for statistical analysis (median and variance). In simulations, the porosity is kept $3 \%$ and $N_{\text {grid }} / l_{c}=20$. Solid network is comprised of $6 \times 6 \times 6$ truncated octahedron grains. (c-e) Visualization of solid liquid interface colored with mean curvature for cases marked in (a). The interface color and color bar show curvature is almost constant in all cases. (f-g) Effect of grid size on mean value and error of $\kappa$ and $\theta$. Due to time intensity of simulations, only four grains are considered in simulations. Finer grid size makes the standard deviation of data smaller but doesn't change mean values.

size ( $\left.\Delta t \propto \Delta x^{4}\right)$, simulations for studying the grid size were limited to four grains. 


\section{Simulation Performance}

Fig. 8a shows the memory usage for two-dimensional simulation comparing memory intensity of interfacial area minimization and the level set method presented here. The computation domain consist of a network of $10 \times 10$ grains. Fig. $8 \mathrm{~b}$ presents the CPU time for same simulations with different grid sizes. Simulation times are orders of magnitude larger for level set method. CFL condition for stability of explicit integration requires $\Delta t<C \Delta x^{4}$ and naturally simulation time grows with slope at least 4 on log-log scale. We were not able to finish simulation with $N_{\text {grid }} / l_{c}>100$.

A comparison between Eq. 5 and Eq. 19 reveals that in two-dimensional simulations, Eq. 5 is an ODE but in the level set method, we solve a nonlinear system of PDE in two-dimensions in order to get one-dimensional interfacial curve (zero-level set). This makes the level set method computationally expensive. Although application of Eqs. 3 and 5 to minimize the interfacial area subject to dihedral angle boundary condition reduces computational cost, tracking topological changes, specially in 3-D, is not trivial. The level set method takes advantages of an implicit form, allowing explicit time integration of highly non-linear terms on a fixed Cartesian grid. In this work, all computations are performed on a single processor (Intel(R) Xeon(R) CPU E3-1270 $3.50 \mathrm{GHz}$ and with $32 \mathrm{~GB}$ RAM).

\section{Simulation Examples and Discussion}

In this section we present three-dimensional simulation results in polycrystalline materials comprised of uniform and non-uniform grains. First set of simulations are done on a network of $6 \times 6 \times 6$ symmetric truncated octahedron grains. Fig 9 shows the distribution

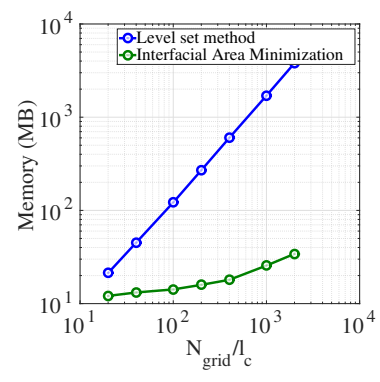

(a)

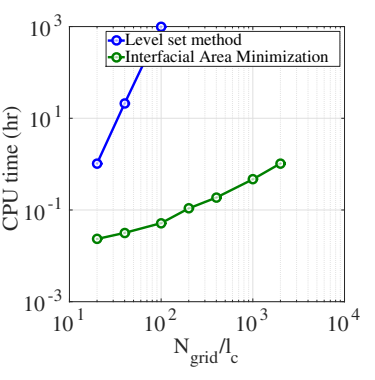

(b)
Figure 8: Effect of grid size on (a) memory usage and (b) CPU time for both level set method and intefacial area minimization problem with identical two-dimensional network of grains. As shown, level set model, by orders of magnitudes, is more computationally expensive. of liquid on grain boundaries for different dihedral angles and porosities. As can be seen, liquid phase is connected along the grain edges when $\theta<60^{\circ}$. Increasing dihedral angle to values above $60^{\circ}$ will result in negative solid-liquid interface curvature and liquid resides in disconnected pockets on grain corners.

In the second set of simulations, we present the distribution of liquid phase in textural equilibrium with a medium comprised of unequal grains. The chosen polycrystalline lattice is a cantitruncated cubic honeycomb which is a uniform space-filling tessellation in threedimensions comprised of truncated cuboctahedra, truncated octahedra, and cubes in a ratio of 1:1:3. The results presented in Fig 10 show the complexity and richness of texturally equilibrated pore networks and confirm that the pore network is percolating in all directions even for very small porosities when $\theta<60^{\circ}$. In the case

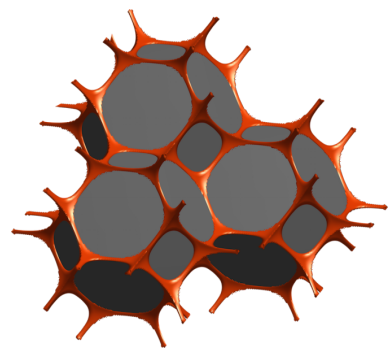

(a) $\phi=1 \%, \theta=10^{\circ}$

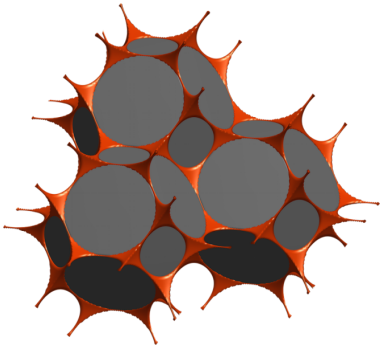

(c) $\phi=1 \%, \theta=60^{\circ}$

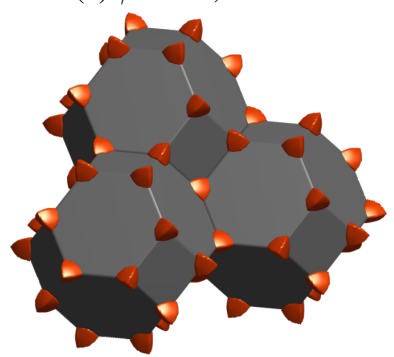

(e) $\phi=1 \%, \theta=90^{\circ}$

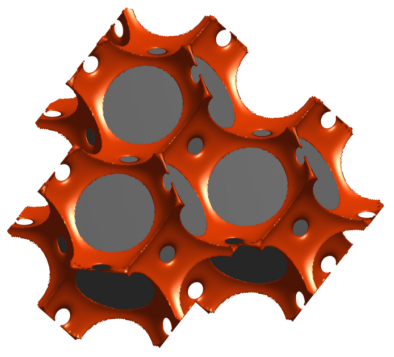

(b) $\phi=4 \%, \theta=10^{\circ}$

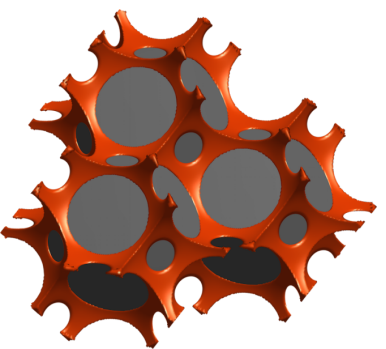

(d) $\phi=4 \%, \theta=60^{\circ}$

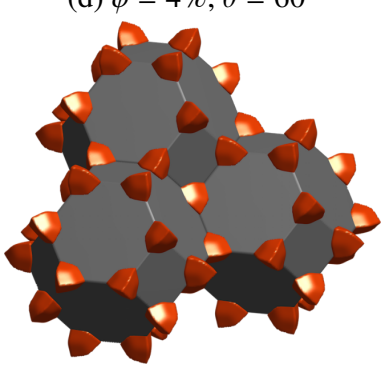

(f) $\phi=4 \%, \theta=90^{\circ}$
Figure 9: Texturally equilibrated pore networks in a polycrystalline solid with an isotropic fabric. Solid network is comprised of $6 \times 6 \times 6$ uniform truncated octahedron grains. 
of $\theta=10^{\circ}$ and $\phi=4 \%$ (Fig. 10b) the liquid spreads wider on smaller grain boundaries (square faces) and grain boundaries are wetted at smaller porosities in comparison to polycrystalline materials composed of equal grains. Consequently, grain boundary wetting is likely to be more common in polycrystalline materials with disordered or unequal grains. An increase in the dihedral angle at constant porosity reduces the wetting tendency of the liquid and texturally equilibrated pore networks form channels along the grain edges in case of $\theta=60^{\circ}$ (Figs. 10c and 10d). Further increase in dihedral angle results in negative curvature of the solidliquid interface and the liquid resides in disconnected pockets on grain corners (Figs. 10e and 10f). Simulation results for a cantitruncated cubic honeycomb lattice shows that the level set method is able to handle

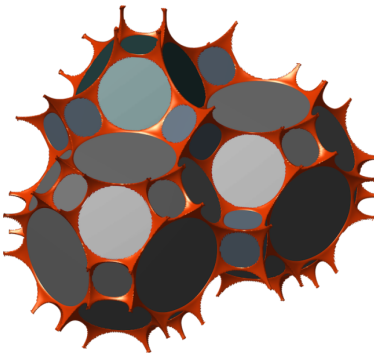

(a) $\phi=1 \%, \theta=10^{\circ}$

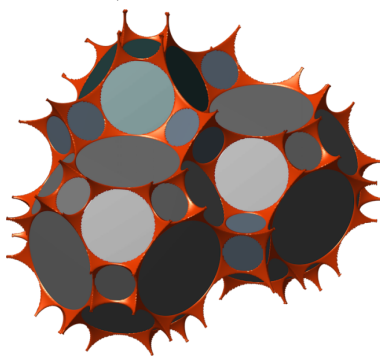

(c) $\phi=1 \%, \theta=60^{\circ}$

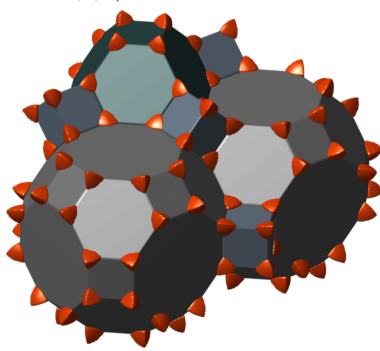

(e) $\phi=1 \%, \theta=90^{\circ}$

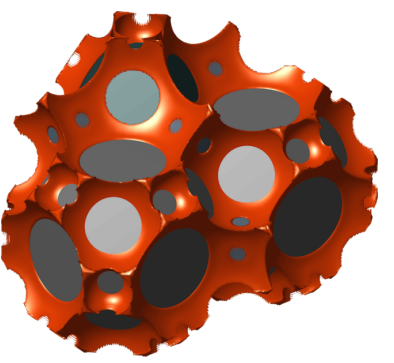

(b) $\phi=4 \%, \theta=10^{\circ}$

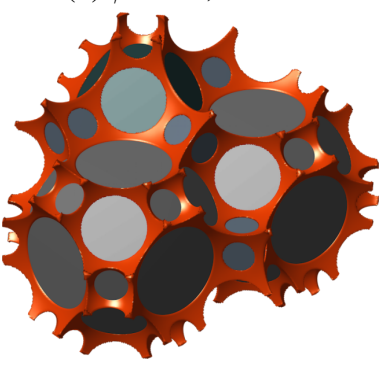

(d) $\phi=4 \%, \theta=60^{\circ}$

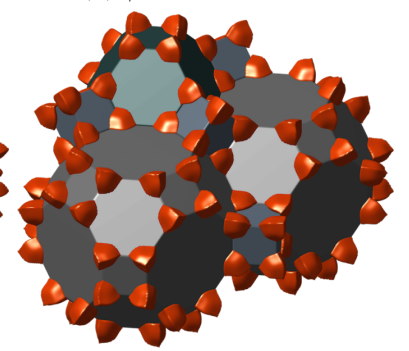

(f) $\phi=4 \%, \theta=90^{\circ}$
Figure 10: Texturally equilibrated pore networks in a polycrystalline solid with unequal grains. Grain configuration is a cantitruncated cubic honeycomb lattice. Results show that the developed model can deal with arbitrary geometries. Displayed images are selected from a domain of 900 grains. the simulation on a polycrystalline solid comprised of grains with arbitrary geometry, as long as the input geometry is space filling and there are two-dimensional solid-solid contact between grains.

\section{Conclusion}

In this work we presented a novel level set model to compute the liquid-solid interface in a porous medium at textural equilibrium. The geometric flexibility of the level set method overcomes the difficulties posed by the complex topology of the interface and allows the simulation in realistic geometries. The computational cost of the numerical method is much higher than previous methods based on explicit computation of the interface. To reduce the cost of the level set method we have introduced the domain decomposition while calculating coupling terms on original computational domain. This results in enormous improvement in computational cost, enabling us to run the simulation for almost 1000 grains on desktop/notebook computer. While high-accuracy numerical discretization allows computations on relatively coarse grids the results have to be interpolated to a fine grid for subsequent visualization. Three dimensional simulation results are presented show the level set approach developed here is applicable to polycrystalline materials comprised of grains with arbitrary shapes.

\section{Nomenclature}

\section{Acknowledgment}

Soheil Ghanbarzadeh is supported by the Statoil Fellows Program at The University of Texas at Austin. Marc Hesse was partially supported by NSF grant EAR CMG-1025321.

\section{References}

[1] N. von Bargen, H. S. Waff, Permeabilities, interfacial areas and curvatures of partially molten systems: results of numerical computations of equilibrium microstructures, Journal of Geophysical Research 91 (B9) (1986) 9261-9276. doi:10.1029/ JB091iB09p09261.

[2] A. W. Rempel, E. D. Waddington, J. S. Wettlaufer, M. G. Worster, Possible displacement of the climate signal in ancient ice by premelting and anomalous diffusion, Nature 411 (6837) (2001) 568-571. doi : 10.1038/35079043.

[3] S. Lewis, M. Holness, Equilibrium halite-H2o dihedral angles: High rock-salt permeability in the shallow crust?, Geology 24 (5) (1996) 431-434. doi:10.1130/0091-7613(1996) $024<0431$ : EHHODA>2 2.3. CO; 2 .

[4] C. S. Smith, Grains, Phases, And Interfaces: An Interpretation Of Microstructure, Transactions of Metallurgical Society of AIME 175 (1948) 15-51. 


\begin{tabular}{ll}
\hline \hline Notation & Description \\
\hline$\phi$ & porosity (\%) \\
$\theta$ & dihedral angle $\left(^{\circ}\right)$ \\
$\kappa$ & mean curvature \\
$\varphi$ & liquid phase level set function \\
$\psi$ & solid phase level set function \\
$\gamma_{s s}$ & surface free energy of solid-solid interface \\
$\gamma_{s l}$ & surface free energy of solid-liquid interface \\
$\vec{n}$ & normal pointing outward \\
$\Delta_{s} \kappa$ & surface Laplacian of curvature \\
$\Theta$ & polar angle \\
$\Phi$ & azimuthal angle, final level set \\
$\delta(\phi)$ & smeared-out delta function \\
$\Delta x$ & grid size \\
$\epsilon$ & interface bandwidth tuning parameter \\
$\epsilon_{1}$ & error threshold for mean curvature \\
$\epsilon_{2}$ & error threshold for dihedral angle \\
$A_{s s}$ & solid-solid interfacial area \\
$A_{s l}$ & solid-liquid interfacial area \\
$D_{\psi_{i}}$ & computational domain for level set $i$ \\
$f$ & elongation factor \\
$H(\phi)$ & smeared-out Heaviside function \\
$l_{c}$ & characteristic length \\
$N_{\text {gird }}$ & number of grid points \\
$r$ & radial distance \\
$S(\phi)$ & sign function \\
$t$ & pseudo time for evolution of level set \\
$V$ & computational domain volume \\
\hline \hline &
\end{tabular}

[5] S. Ghanbarzadeh, M. Prodanovic, M. A. Hesse, Percolation and Grain Boundary Wetting in Anisotropic Texturally Equilibrated Pore Networks, Phys. Rev. Lett. 113 (4) (2014) 048001.

[6] M. Holness, B, Decoding dihedral angles in melt-bearing and solidified rocks. In: (Eds.) M.A. Forster and John D. Fitz Gerald, Journal of the Virtual Explorer 35 (2010) paper 2. doi : 10.3809/jvirtex. 2011.00265 .

[7] C. S. Smith, Some Elementary Principles of Polycrystalline Microstructure, Metallurgical Reviews 9 (1) (1964) 1-48.

[8] P. G. de Gennes, Wetting: statics and dynamics, Reviews of Modern Physics 57 (3) (1985) 827-863. doi:10.1103/ RevModPhys .57.827.

[9] J. R. Bulau, H. S. Waff, J. A. Tyburczy, Mechanical and Thermodynamic Constraints on Fluid Distribution in Partial Melts, Journal of Geophysical Research 84 (1979) 6102-6108. doi : 10.1029/JB084iB11p06102.

[10] S. C. van der Marck, Evidence for a nonzero tansport threshold in porous media, Water Resources Research 35 (2) (1999) 595599. doi:10.1029/1998WR900062.

[11] W. Zhu, G. A. Gaetani, F. Fusseis, L. G. J. Montesi, F. D. Carlo, Microtomography of Partially Molten Rocks: ThreeDimensional Melt Distribution in Mantle Peridotite, Science 332 (6025) (2011) 88-91. doi:10.1126/science. 1202221.

[12] J. Schoenherr, J. L. Urai, P. A. Kukla, R. Littke, Z. Schlder, J.-M. Larroque, M. J. Newall, N. Al-Abry, H. A. Al-Siyabi, Z. Rawahi, Limits to the sealing capacity of rock salt: A case study of the infra-Cambrian Ara Salt from the South Oman salt basin, AAPG Bulletin 91 (11) (2007) 1541-1557. doi: 10.1306/06200706122.

[13] A. V. Sobolev, N. Shimizu, Superdepleted melts and ocean mantle permeability, Transactions Doklady-Russian Academy of Sciences Earth Science Sections 328 (1993) 182-182.

[14] C. C. Lundstrom, J. Gill, Q. Williams, M. R. Perfit, Mantle Melting and Basalt Extraction by Equilibrium Porous Flow, Science 270 (1995) 1958-1961. doi:10.1126/science.270.5244. 1958.

[15] M. Spiegelman, T. Elliott, Consequences of melt transport for uranium series disequilibrium in young lavas, Earth and Planetary Science Letters 118 (1993) 1-20. doi:10.1016/ 0012-821X (93) 90155-3.

[16] M. B. Holness, S. Lewis, The structure of the halite-brine interface inferred from pressure and temperature variations of equilibrium dihedral angles in the halite- $\mathrm{H} 2 \mathrm{o}-\mathrm{CO} 2$ system, Geochimica et Cosmochimica Acta 61 (4) (1997) 795-804. doi:10.1016/S0016-7037(96) 00370-5.

[17] M. W. Downey, Evaluating seals for hydrocarbon accumulations, AAPG Bulletin 68 (11) (1984) 1752-1763.

[18] W. Beere, A unifying theory of the stability of penetrating liquid phases and sintering pores, Acta Metallurgica 23 (1) (1975) 131-138. doi: 10.1016/0001-6160 (75) 90078-4.

[19] Y. Takei, Effect of pore geometry on VP/VS: From equilibrium geometry to crack, Journal of Geophysical Research: Solid Earth 107 (B2) (2002) ECV6 1-ECV6 12. doi:10.1029/ 2001 JB000522.

[20] M. Pervukhina, Y. Kuwahara, Correlations between electrical and elastic properties of solidliquid composites with interfacial energy-controlled equilibrium microstructures, Earth and Planetary Science Letters 265 (34) (2008) 410-422. doi : 10.1016/ j.epsl.2007.10.038.

[21] J. Wimert, S. Hier-Majumder, A three-dimensional microgeodynamic model of melt geometry in the Earth's deep interior, Journal of Geophysical Research: Solid Earth 117 (B4) (2012) B04203. doi: 10.1029/2011JB009012.

[22] J. Oprea, The Mathematics of Soap Films: Explorations With Maple (Student Mathematical Library, Vol. 10), Amer Mathematical Society, 2000.

[23] J. F. Nye, The Geometry of Water Veins and Nodes in Polycrystalline Ice, Journal of Glaciology 35 (119) (1989) 17-22. doi:10.3189/002214389793701437.

[24] M. J. Cheadle, Properties of texturally equilibrated two-phase aggregates., Ph.D., University of Cambridge (1989).

[25] G. Hirth, D. L. Kohlstedt, Experimental constraints on the dynamics of the partially molten upper mantle: Deformation in the diffusion creep regime, Journal of Geophysical Research: Solid Earth 100 (B2) (1995) 1981-2001. doi:10.1029/94JB02128.

[26] A. L. Endres, T. Murray, A. D. Booth, L. J. West, A new framework for estimating englacial water content and pore geometry using combined radar and seismic wave velocities, Geophysical Research Letters 36 (4) (2009) L04501. doi:10.1029/ 2008GL036876.

[27] S. Osher, J. A. Sethian, Fronts propagating with curvaturedependent speed: Algorithms based on Hamilton-Jacobi formulations, Journal of Computational Physics 79 (1) (1988) 12-49. doi:10.1016/0021-9991(88)90002-2.

[28] S. J. Osher, R. P. Fedkiw, Level Set Methods and Dynamic Implicit Surfaces, Springer, 2002.

[29] J. A. Sethian, Level set methods and fast marching methods, 2nd Edition, Cambridge University Press, Cambridge, 1999.

[30] D. Peng, B. Merriman, S. Osher, H. Zhao, M. Kang, A PDEBased Fast Local Level Set Method, Journal of Computational Physics 155 (2) (1999) 410-438. doi:10.1006/jcph. 1999. 6345. 
[31] P. Smereka, Semi-Implicit Level Set Methods for Curvature and Surface Diffusion Motion, Journal of Scientific Computing 19 (1-3) (2003) 439-456. doi : 10.1023/A : 1025324613450.

[32] P. D. M. Spelt, A level-set approach for simulations of flows with multiple moving contact lines with hysteresis, Journal of Computational Physics 207 (2) (2005) 389-404. doi:10. $1016 / j \cdot j c p .2005 .01 .016$.

[33] H. Liu, S. Krishnan, S. Marella, H. S. Udaykumar, Sharp interface Cartesian grid method II: A technique for simulating droplet interactions with surfaces of arbitrary shape, Journal of Computational Physics 210 (1) (2005) 32-54. doi : 10.1016/ j.jcp.2005.03.032.

[34] Z. Li, M.-C. Lai, G. He, H. Zhao, An augmented method for free boundary problems with moving contact lines, Computers \& Fluids 39 (6) (2010) 1033-1040. doi:10.1016/j. compfluid.2010.01.013.

[35] E. Jettestuen, J. O. Helland, M. Prodanovic, A level set method for simulating capillary-controlled displacements at the pore scale with nonzero contact angles, Water Resources Research 49 (8) (2013) 4645-4661. doi : 10 . 1002/wrcr . 20334.

[36] M. Prodanovic, S. L. Bryant, A level set method for determining critical curvatures for drainage and imbibition, Journal of Colloid and Interface Science 304 (2) (2006) 442-458. doi: 10.1016/j.jcis.2006.08.048.

[37] W. Lee, G. Son, J. J. Jeong, Numerical Analysis of Bubble Growth and Departure from a Microcavity, Numerical Heat Transfer, Part B: Fundamentals 58 (5) (2010) 323-342. doi : 10.1080/10407790.2010.522871.

[38] D. L. Chopp, J. A. Sethian, Motion by Intrinsic Laplacian of Curvature, Interfaces and Free Boundaries 1 (1999) 1-18.

[39] I. M. Mitchell, The Flexible, Extensible and Efficient Toolbox of Level Set Methods, Journal of Scientific Computing 35 (2-3) (2008) 300-329. doi : 10.1007/s10915-007-9174-4.

[40] W. E. Lorensen, H. E. Cline, Marching cubes: A high resolution 3d surface construction algorithm, in: SIGGRAPH 1987, Vol. 21 of 4, Anaheim, CA, USA, 1987, pp. 163-169. doi: 10.1145/37402.37422.

[41] R. T. Armstrong, M. L. Porter, D. Wildenschild, Linking porescale interfacial curvature to column-scale capillary pressure, Advances in Water Resources 46 (2012) 55-62. doi : 10.1016/ j.advwatres.2012.05.009. 